\title{
Algebras of finitary relations
}

\author{
V P Tsvetov ${ }^{1}$ \\ ${ }^{1}$ Samara National Research University, Moskovskoe Shosse 34A, Samara, Russia, 443086 \\ e-mail: tsf-su@mail.ru
}

\begin{abstract}
Algebras of finitary relations naturally generalize the algebra of binary relations with the left composition. In this paper, we consider some properties of such algebras. It is well known that we can study the hypergraphs as finitary relations. In this way the results can be applied to graph and hypergraph theory, automatons and artificial intelligence.
\end{abstract}

\section{Introduction}

It is obvious that graphs and binary relations are closely related. We often use the facts of the binary relations theory in graph theory to solve some algorithmic problems. In the same way, we can consider hypergraphs as finitary relations. This could be a good idea for IT and AI, especially for pattern recognition and machine learning [1-13].

By now it has become common to use universal algebras [14] in various applications [15]. Algebraic methods can also be efficiently applied in graph theory. For example, the shortest path problem can be solved by transitive closure algorithm for binary relation [16].

In this way, and following by [17], we are going to study hypergraphs as elements of algebraic structures.

At first, we define a (n-uniform) hypergraph as a finitary relation on finite set $U$, in other words, as a subset of $U^{n}$. In case of $n=2$ this leads to graph as a binary relation. Boolean algebras $\left\langle 2^{U \times U},\left(\cup, \cap,{ }^{-}, \varnothing, U \times U\right)\right\rangle$ and $\left\langle 2^{U^{n}},\left(\cup, \cap,-\varnothing, U^{n}\right)\right\rangle$ are well known to us.

It is less trivial to define the inverse operation and the left composition for finitary relations. We have to start from inverse operation, left and right compositions for binary relations:

$$
\begin{gathered}
R^{-1}=\left\{\left(u_{2}, u_{1}\right) \mid\left(u_{1}, u_{2}\right) \in R\right\}, \\
R_{1} \circ R_{2}=\left\{\left(u_{1}, u_{2}\right) \mid \exists u_{0}\left(u_{1}, u_{0}\right) \in R_{1} \wedge\left(u_{0}, u_{2}\right) \in R_{2}\right\}, \\
R_{1} \circ R_{2}=R_{2} \circ R_{1}=\left\{\left(u_{1}, u_{2}\right) \mid \exists u_{0}\left(u_{0}, u_{2}\right) \in R_{1} \wedge\left(u_{1}, u_{0}\right) \in R_{2}\right\}
\end{gathered}
$$

Note that $\left\langle 2^{U_{\times} U},\left({ }^{\circ}, I\right)\right\rangle \sim\left\langle\mathrm{R}^{U_{\times} U},(\cdot, I)\right\rangle$ are isomorphic monoids, where $I$ is identity relation on $U$. By the way, we can define operations

$$
\begin{aligned}
& R_{1} \circ{ }_{1} R_{2}=R_{1}^{-1} \circ R_{2}, \\
& R_{1} \bullet R_{2}=R_{2} \circ R_{1}, \\
& R_{1} \circ_{2} R_{2}=R_{1} \circ R_{2}^{-1},
\end{aligned}
$$




$$
\begin{aligned}
& R_{1} \bullet_{2} R_{2}=R_{2} \circ R_{1}^{-1}, \\
& R_{1} \circ_{3} R_{2}=R_{1}^{-1} \circ R_{2}^{-1}, \\
& R_{1} \bullet_{3} R_{2}=R_{2}^{-1} \circ R_{1}^{-1} .
\end{aligned}
$$

This makes it possible to set the following pairs of isomorphic magmas. $\left\langle 2^{U \times U},\left(\circ_{1}, I\right)\right\rangle \square\left\langle 2^{U \times U},\left(\square_{1}, I\right)\right\rangle$ are isomorphic magmas with left identity elements. $\left\langle 2^{U \times U},\left(\circ_{2}, I\right)\right\rangle \square\left\langle 2^{U \times U},\left(\square_{2}, I\right)\right\rangle$ are isomorphic magmas with right identity elements. $\left\langle 2^{U \times U},\left(\circ_{3}\right)\right\rangle \square\left\langle 2^{U \times U},\left(\square_{3}\right)\right\rangle$ are isomorphic magmas without identity elements.

It is easy to see that in the symmetric case $R=R^{-1}$ all of monogenic monoids $\left\langle\left\{R^{n}\right\}_{n=0}^{\infty},(\circ, I)\right\rangle$, $\left\langle\left\{R^{n}\right\}_{n=0}^{\infty},(\square, I)\right\rangle,\left\langle\left\{R^{n}\right\}_{n=0}^{\infty},\left(\circ_{i}, I\right)\right\rangle,\left\langle\left\{R^{n}\right\}_{n=0}^{\infty},(\square, I)\right\rangle(i \in 1 . .3)$ are equal.

The monogenic monoid $\left\langle\left\{R^{n}\right\}_{n=0}^{\infty},(\circ, I)\right\rangle$ and distributive algebraic structure $\left\langle\left\{R^{n}\right\}_{n=0}^{\infty},(\circ, \cup, I, \varnothing)\right\rangle$ are useful to treat all-pairs shortest path problem [16]. We are going to define and study hypergraph operations similar to (1)-(9).

\section{Algebras of finitary relations}

Let us consider the underlying set of finitary relations $2^{U^{n}}$, and define the following unary and binary operations for $i \neq j$

$$
\begin{gathered}
R^{(\mathrm{ij})}=R^{(\mathrm{ji})}=\left\{\left(u_{1}, . ., u_{j}, . ., u_{i}, . ., u_{n}\right) \mid\left(u_{1}, . ., u_{i}, . ., u_{j}, . ., u_{n}\right) \in R\right\}, \\
R_{1} \circ_{i j} R_{2}=\left\{\left(u_{1}, . ., u_{i}, . ., u_{j}, . ., u_{n}\right) \mid \exists u_{0}\left(u_{1}, . ., u_{0}, \ldots, u_{j}, \ldots, u_{n}\right) \in R_{1} \wedge\left(u_{1}, . ., u_{i}, \ldots, u_{0}, \ldots, u_{n}\right) \in R_{2}\right\} .
\end{gathered}
$$

Obviously, the operation (10) is an involution.

$$
\left(R^{(\mathrm{ij})}\right)^{(\mathrm{ij})}=R .
$$

Moreover,

$$
R_{1} \circ{ }_{i j} R_{2}=R_{2} \circ{ }_{j i} R_{1} .
$$

It is easy to prove that operation (11) is associative. Actually, $\left(u_{1}, . ., u_{i}, . ., u_{j}, . ., u_{n}\right) \in R_{1} \circ_{i j}\left(R_{2} \circ_{i j} R_{3}\right) \Leftrightarrow \exists u_{0}\left(u_{1}, . ., u_{0}, \ldots, u_{j}, . ., u_{n}\right) \in R_{1} \wedge\left(u_{1}, . ., u_{i}, . ., u_{0}, . ., u_{n}\right) \in R_{2} \circ_{i j} R_{3} \Leftrightarrow$ $\Leftrightarrow \exists u_{0}\left(u_{1}, . ., u_{0}, . ., u_{j}, . ., u_{n}\right) \in R_{1} \wedge\left(\exists u_{0}^{\prime}\left(u_{1}, . ., u_{0}^{\prime}, . ., u_{0}, . ., u_{n}\right) \in R_{2} \wedge\left(u_{1}, . ., u_{i}, . ., u_{0}^{\prime}, . ., u_{n}\right) \in R_{3}\right) \Leftrightarrow$ $\Leftrightarrow \exists u_{0}^{\prime}\left(\exists u_{0}\left(u_{1}, . ., u_{0}, . ., u_{j}, . ., u_{n}\right) \in R_{1} \wedge\left(u_{1}, . ., u_{0}^{\prime}, . ., u_{0}, . ., u_{n}\right) \in R_{2}\right) \wedge\left(u_{1}, . ., u_{i}, . ., u_{0}^{\prime}, \ldots, u_{n}\right) \in R_{3} \Leftrightarrow$ $\Leftrightarrow \exists u_{0}^{\prime}\left(u_{1}, \ldots, u_{0}^{\prime}, \ldots, u_{j}, . ., u_{n}\right) \in R_{1} \circ_{i j} R_{2} \wedge\left(u_{1}, . ., u_{i}, . ., u_{0}^{\prime}, \ldots, u_{n}\right) \in R_{3} \Leftrightarrow$ $\Leftrightarrow\left(u_{1}, . ., u_{i}, . ., u_{j}, . ., u_{n}\right) \in\left(R_{1} \circ{ }_{i j} R_{2}\right) \circ_{i j} R_{3}$.

Then we set

It is easy to see

$$
I_{i j}=\left\{\left(u_{1}, . ., u_{i}, . ., u_{j}, . ., u_{n}\right) \mid k \in 1 . . n \wedge u_{k} \in U \wedge u_{j}=u_{i}\right\} \in 2^{U^{n}}
$$

$$
\begin{aligned}
& \left(u_{1}, . ., u_{i}, . ., u_{j}, . ., u_{n}\right) \in I_{i j} \circ_{i j} R \Leftrightarrow \exists u_{0}\left(u_{1}, . ., u_{0}, . ., u_{j}, . ., u_{n}\right) \in I_{i j} \wedge\left(u_{1}, . ., u_{i}, \ldots, u_{0}, . ., u_{n}\right) \in R \Leftrightarrow \\
& \Leftrightarrow \exists u_{0}\left(u_{1}, . ., u_{i}, . ., u_{0}, . ., u_{n}\right) \in R \wedge u_{j}=u_{0} \Leftrightarrow\left(u_{1}, . ., u_{i}, . ., u_{j}, . ., u_{n}\right) \in R,
\end{aligned}
$$

and similarly

$$
\begin{aligned}
& \left(u_{1}, . ., u_{i}, . ., u_{j}, . ., u_{n}\right) \in R \circ_{i j} I_{i j} \Leftrightarrow \exists u_{0}\left(u_{1}, . ., u_{0}, . ., u_{j}, . ., u_{n}\right) \in R \wedge\left(u_{1}, . ., u_{i}, . ., u_{0}, . ., u_{n}\right) \in I_{i j} \Leftrightarrow \\
& \Leftrightarrow \exists u_{0}\left(u_{1}, . ., u_{0}, . ., u_{j}, . ., u_{n}\right) \in R \wedge u_{i}=u_{0} \Leftrightarrow\left(u_{1}, . ., u_{i}, . ., u_{j}, . ., u_{n}\right) \in R .
\end{aligned}
$$

Thus, 
Hence we have just proved the

$$
I_{i j} \circ_{i j} R=R \circ_{i j} I_{i j}=R \text {. }
$$

Lemma 1. $\left\langle 2^{U^{n}},\left(o_{i j}, I_{i j}\right)\right\rangle$ is a monoid.

Note that

$$
\begin{aligned}
& \left(u_{1}, . ., u_{i}, . ., u_{j}, . ., u_{n}\right) \in\left(R_{1} \circ_{i j} R_{2}\right)^{(\mathrm{ij})} \Leftrightarrow\left(u_{1}, . ., u_{j}, . ., u_{i}, . ., u_{n}\right) \in R_{1} \circ_{i j} R_{2} \Leftrightarrow \\
& \Leftrightarrow \exists u_{0}\left(u_{1}, . ., u_{0}, . ., u_{i}, . ., u_{n}\right) \in R_{1} \wedge\left(u_{1}, . ., u_{j}, . ., u_{0}, . ., u_{n}\right) \in R_{2} \Leftrightarrow \\
& \Leftrightarrow \exists u_{0}\left(u_{1}, . ., u_{i}, . ., u_{0}, . ., u_{n}\right) \in R_{1}^{(\mathrm{ij})} \wedge\left(u_{1}, . ., u_{0}, . ., u_{j}, . ., u_{n}\right) \in R_{2}^{(\mathrm{ij})} \Leftrightarrow \\
& \Leftrightarrow\left(u_{1}, . ., u_{i}, . ., u_{j}, . ., u_{n}\right) \in R_{1}^{(\mathrm{jij})} \circ_{j i} R_{2}^{(\mathrm{jij})} \Leftrightarrow\left(u_{1}, . ., u_{i}, . ., u_{j}, . ., u_{n}\right) \in R_{2}^{(\mathrm{ij})} \circ_{i j} R_{1}^{(\mathrm{ij})} .
\end{aligned}
$$

In that way

$$
\left(R_{1} \circ_{i j} R_{2}\right)^{(\mathrm{ij})}=R_{1}^{(\mathrm{ij})} \circ_{j i} R_{2}^{(\mathrm{ij})}=R_{2}^{(\mathrm{ij})} \circ_{i j} R_{1}^{(\mathrm{ij})} .
$$

Hence the bijective function $f(R):=R^{(\mathrm{ij})}$ is an isomorphism of monoids $\left\langle 2^{U^{n}},\left(\circ_{i j}, I_{i j}\right)\right\rangle$ and $\left\langle 2^{U^{n}},\left(\circ_{j i}, I_{j i}\right)\right\rangle$.

\section{Moreover,}

$$
\begin{aligned}
& \left(u_{1}, . ., u_{i}, . ., u_{k}, . ., u_{j}, . ., u_{n}\right) \in\left(R_{1} \circ_{i k} R_{2}\right)^{(\mathrm{ij})} \Leftrightarrow\left(u_{1}, . ., u_{j}, . ., u_{k}, . ., u_{i}, . ., u_{n}\right) \in R_{1} \circ_{i k} R_{2} \Leftrightarrow \\
& \Leftrightarrow \exists u_{0}\left(u_{1}, . ., u_{0}, . ., u_{k}, . ., u_{i}, . ., u_{n}\right) \in R_{1} \wedge\left(u_{1}, . ., u_{j}, . ., u_{0}, . ., u_{i}, . ., u_{n}\right) \in R_{2} \Leftrightarrow \\
& \Leftrightarrow \exists u_{0}\left(u_{1}, . ., u_{i}, . ., u_{k}, . ., u_{0}, . ., u_{n}\right) \in R_{1}^{(\mathrm{ij})} \wedge\left(u_{1}, . ., u_{i}, . ., u_{0}, . ., u_{j}, . ., u_{n}\right) \in R_{2}^{(\mathrm{ij})} \Leftrightarrow \\
& \Leftrightarrow\left(u_{1}, . ., u_{i}, \ldots, u_{j}, . ., u_{n}\right) \in R_{1}^{(\mathrm{jij})} \circ_{j k} R_{2}^{(\mathrm{ij})} \Leftrightarrow\left(u_{1}, . ., u_{i}, . ., u_{j}, . ., u_{n}\right) \in R_{2}^{(\mathrm{ij})} \circ_{k j} R_{1}^{(\mathrm{ij})} .
\end{aligned}
$$

From which we obtain

Hence we have proved the

$$
\left(R_{1} \circ_{i k} R_{2}\right)^{(\mathrm{ij})}=R_{1}^{(\mathrm{ij})} \circ_{j k} R_{2}^{(\mathrm{ij})}=R_{2}^{(\mathrm{ij})} \circ_{k j} R_{1}^{(\mathrm{ij})} .
$$

Lemma 2. Monoids $\left\langle 2^{U^{n}},\left(\circ_{i k}, I_{i k}\right)\right\rangle$ and $\left\langle 2^{U^{n}},\left({ }_{j k}, I_{j k}\right)\right\rangle$ are isomorphic, as well as monoids $\left\langle 2^{U^{n}},\left(o_{i j}, I_{i j}\right)\right\rangle$ and $\left\langle 2^{U^{n}},\left(o_{j i}, I_{j i}\right)\right\rangle$.

Let us set an algebraic structure $\left\langle 2^{U^{n}},\left({ }_{i j}, \circ_{i k}, I_{i j}, I_{i k}\right)\right\rangle$ and then we can write the following logical consequences:

$$
\begin{aligned}
& \left(u_{1}, \ldots, u_{i}, . ., u_{j}, \ldots, u_{k}, . ., u_{n}\right) \in R_{1} \circ_{i j}\left(R_{2} \circ_{i k} R_{3}\right) \Leftrightarrow \exists u_{0}\left(u_{1}, \ldots, u_{0}, . ., u_{j}, . ., u_{k}, . ., u_{n}\right) \in R_{1} \wedge \\
& \wedge\left(u_{1}, . ., u_{i}, \ldots, u_{0}, . ., u_{k}, . ., u_{n}\right) \in R_{2} \circ_{i k} R_{3} \Leftrightarrow \exists u_{0} \exists u_{0}^{\prime}\left(u_{1}, . ., u_{0}, . ., u_{j}, . ., u_{k}, . ., u_{n}\right) \in R_{1} \wedge \\
& \wedge\left(u_{1}, \ldots, u_{0}^{\prime}, \ldots, u_{0}, \ldots, u_{k}, . ., u_{n}\right) \in R_{2} \wedge\left(u_{1}, \ldots, u_{i}, \ldots, u_{0}, \ldots, u_{0}^{\prime}, . ., u_{n}\right) \in R_{3} \Leftrightarrow \\
& \exists u_{0}^{\prime} \exists u_{0}\left(u_{1}, . ., u_{0}, . ., u_{j}, . ., u_{k}, . ., u_{n}\right) \in R_{1} \wedge\left(u_{1}, . ., u_{0}^{\prime}, . ., u_{0}, . ., u_{k}, . ., u_{n}\right) \in R_{2} \wedge \\
& \wedge\left(u_{1}, . ., u_{i}, . ., u_{0}, . ., u_{0}^{\prime}, . ., u_{n}\right) \in R_{3} \Rightarrow \\
& \exists u_{0}^{\prime}\left(\exists u_{0}\left(u_{1}, . ., u_{0}, . ., u_{j}, . ., u_{k}, . ., u_{n}\right) \in R_{1} \wedge\left(u_{1}, . ., u_{0}^{\prime}, . ., u_{0}, . ., u_{k}, . ., u_{n}\right) \in R_{2}\right) \wedge \\
& \wedge\left(\exists u_{0}\left(u_{1}, . ., u_{i}, . ., u_{0}, . ., u_{0}^{\prime}, . ., u_{n}\right) \in R_{3}\right) \Leftrightarrow \exists u_{0}^{\prime}\left(u_{1}, . ., u_{0}^{\prime}, \ldots, u_{j}, . ., u_{k}, . ., u_{n}\right) \in R_{1} \circ_{i j} R_{2} \wedge \\
& \wedge\left(\exists u_{0}\left(u_{1}, . ., u_{i}, . ., u_{0}, . ., u_{0}^{\prime}, . ., u_{n}\right) \in R_{3} \wedge\left(u_{1}, . ., u_{0}, . ., u_{j}, . ., u_{0}^{\prime}, . ., u_{n}\right) \in 1_{R}\right) \Leftrightarrow \\
& \Leftrightarrow \exists u_{0}^{\prime}\left(u_{1}, . ., u_{0}^{\prime}, . ., u_{j}, . ., u_{k}, . ., u_{n}\right) \in R_{1} \circ_{i j} R_{2} \wedge\left(u_{1}, . ., u_{i}, . ., u_{j}, . ., u_{0}^{\prime}, . ., u_{n}\right) \in R_{3} \circ_{i j} 1_{R} \Leftrightarrow \\
& \Leftrightarrow\left(u_{1}, \ldots, u_{i}, . ., u_{j}, \ldots, u_{k}, \ldots, u_{n}\right) \in\left(R_{1} \circ{ }_{i j} R_{2}\right) \circ_{i k}\left(R_{3} \circ{ }_{i j} 1_{R}\right) \text {. }
\end{aligned}
$$

This means that the following Lemma is true. 
Lemma 3. In an ordered algebra $\left\langle 2^{U^{n}},\left(\circ_{i j}, \circ_{i k}, \subseteq, I_{i j}, I_{i k}, 0_{R}, 1_{R}\right)\right\rangle$, the pseudo distributive law holds

$$
R_{1} \circ \circ_{i j}\left(R_{2} \circ{ }_{i k} R_{3}\right) \subseteq\left(R_{1} \circ{ }_{i j} R_{2}\right) \circ_{i k}\left(R_{3} \circ{ }_{i j} 1_{R}\right) .
$$

According to [17], we use the notation $1_{R}:=U^{n}$ and $0_{R}:=\varnothing$.

Then look at composition

$$
\begin{aligned}
& \left(u_{1}, \ldots, u_{i}, \ldots, u_{j}, \ldots, u_{n}\right) \in R \circ_{i j} R^{(\mathrm{ij})} \Leftrightarrow \exists u_{0}\left(u_{1}, \ldots, u_{0}, \ldots, u_{j}, . ., u_{n}\right) \in R \wedge\left(u_{1}, \ldots, u_{i}, \ldots, u_{0}, \ldots, u_{n}\right) \in R^{(\mathrm{ij})} \Leftrightarrow \\
& \Leftrightarrow \exists u_{0}\left(u_{1}, \ldots, u_{0}, \ldots, u_{j}, . ., u_{n}\right) \in R \wedge\left(u_{1}, \ldots, u_{0}, . ., u_{i}, . ., u_{n}\right) \in R .
\end{aligned}
$$

Definition 1. The finitary relation $R$ is called a function from $i$-th to $j$-th argument if

$$
\forall u_{1}, \ldots, u_{i}, . ., u_{j}, u_{j}^{\prime}, . ., u_{n}\left(u_{1}, . ., u_{i}, . ., u_{j}, . ., u_{n}\right) \in R \wedge\left(u_{1}, . ., u_{i}, . ., u_{j}^{\prime}, . ., u_{n}\right) \in R \rightarrow u_{j}=u_{j}^{\prime} .
$$

We can obtain from (19) - (20) the following set inclusion

$$
\left(u_{1}, . ., u_{i}, . ., u_{j}, . ., u_{n}\right) \in R \circ_{i j} R^{(\mathrm{ij})} \Rightarrow u_{i}=u_{j} \Leftrightarrow\left(u_{1}, . ., u_{i}, . ., u_{j}, . ., u_{n}\right) \in I_{i j} \Leftrightarrow R \circ_{i j} R^{(\mathrm{ij})} \subseteq I_{i j} .
$$

Definition 2. The finitary relation $R$ is called a surjection from $i$-th argument if

$$
\forall u_{1}, \ldots, u_{i-1}, u_{i+1}, . ., u_{j}, . ., u_{n} \exists u_{0}\left(u_{1}, . ., u_{0}, . ., u_{j}, . ., u_{n}\right) \in R \text {. }
$$

From (21) - (22) we can get the reverse set inclusion

$$
I_{i j} \subseteq R \circ_{i j} R^{(\mathrm{ij})} .
$$

Thus, in the case of $R$ is a surjective function from $i$-th to $j$-th argument we have the equality

$$
R \circ{ }_{i j} R^{(\mathrm{ij})}=I_{i j} .
$$

Similarly, in the case of $R$ is a surjective function from $j$-th to $i$-th argument we have the equality

$$
R^{(\mathrm{ij})} \circ_{i j} R=I_{i j} \text {. }
$$

Let us denote the set of surjective functions from both ( $i$-th to $j$-th and $j$-th to $i$-th) arguments as $F_{i j}$. It is easy that $F_{i j}$ is closed by ${ }_{i j}$, and hence we have proved the

Lemma 4. $\left\langle F_{i j},\left(\circ_{i j}, I_{i j}\right)\right\rangle$ is a subgroup of the monoid $\left\langle 2^{U^{n}},\left(\circ_{i j}, I_{i j}\right)\right\rangle$.

As well as binary relations, finitary relations have the following properties [17]

$$
\begin{aligned}
& R_{1} \circ_{i j}\left(R_{2} \cup R_{3}\right)=\left(R_{1} \circ_{i j} R_{2}\right) \cup\left(R_{1} \circ_{i j} R_{3}\right), \\
& \left(R_{2} \cup R_{3}\right) \circ_{i j} R_{1}=\left(R_{2} \circ_{i j} R_{1}\right) \cup\left(R_{3} \circ_{i j} R_{1}\right), \\
& R_{1} \circ_{i j}\left(R_{2} \cap R_{3}\right) \subseteq\left(R_{1} \circ_{i j} R_{2}\right) \cap\left(R_{1} \circ_{i j} R_{3}\right), \\
& \left(R_{2} \cap R_{3}\right) \circ_{i j} R_{1} \subseteq\left(R_{2} \circ_{i j} R_{1}\right) \cap\left(R_{3} \circ_{i j} R_{1}\right),
\end{aligned}
$$

and so we can set an algebraic structures $\left\langle F_{i j},\left(\circ_{i j}, I_{i j}\right)\right\rangle,\left\langle 2^{U^{n}},\left(\cup, \cap, \circ_{i j}, \circ_{i k}{ }^{(i j)}, \subseteq, 0_{R}, 1_{R}, I_{i j}, I_{i k}\right)\right\rangle$ that have properties (12)-(18), (24)-(29).

\section{Conclusion and examples}

We have defined algebraic structures of finitary relations as a common case of well-known algebraic structures of binary relations. We have considered the algebraic structures on an underlying set $2^{U^{n}}$ and sometimes called a finitary relation $R \in 2^{U^{n}}$ by a ( $n$-uniform) hypergraph. The operation $\circ_{i j}$ can be called the "straightening the edges" or "deleting shared intermediate vertices". Let us take an example.

Example 1 (algebraic). Let us set $U=\left\{u_{0}, u_{1}, u_{2}, u_{3}\right\}, \quad\left\langle 2^{U^{3}},\left(\circ_{23}, I_{23}\right)\right\rangle, \quad$ and $R=\left\{\left(u_{1}, u_{0}, u_{3}\right),\left(u_{1}, u_{2}, u_{0}\right)\right\}$. Now we can get 


$$
\begin{gathered}
I_{23}=\left\{\begin{array}{c}
\left(u_{0}, u_{0}, u_{0}\right),\left(u_{0}, u_{1}, u_{1}\right),\left(u_{0}, u_{2}, u_{2}\right),\left(u_{0}, u_{3}, u_{3}\right), \\
\left(u_{1}, u_{0}, u_{0}\right),\left(u_{1}, u_{1}, u_{1}\right),\left(u_{1}, u_{2}, u_{2}\right),\left(u_{1}, u_{3}, u_{3}\right), \\
\left(u_{2}, u_{0}, u_{0}\right),\left(u_{2}, u_{1}, u_{1}\right),\left(u_{2}, u_{2}, u_{2}\right),\left(u_{2}, u_{3}, u_{3}\right), \\
\left(u_{3}, u_{0}, u_{0}\right),\left(u_{3}, u_{1}, u_{1}\right),\left(u_{3}, u_{2}, u_{2}\right),\left(u_{3}, u_{3}, u_{3}\right)
\end{array}\right\}, \\
R \circ_{23} R=\left\{\left(u_{1}, u_{2}, u_{3}\right)\right\}, \\
R \circ_{23} R \circ_{23} R=\varnothing .
\end{gathered}
$$

Despite its simplicity, operation ${ }_{23}$ has some interesting applications. In examples 2, 3 we are going to denote 3-tuple $\left(u_{i_{1}}, u_{i_{2}}, u_{i_{3}}\right)$ as a word $u_{i_{1}} u_{i_{2}} u_{i_{3}}$.

Example 2 (feature selection). Let $R=\left\{u_{1} u_{0} u_{0}, u_{1} u_{1} u_{0}, u_{1} u_{2} u_{0}, u_{1} u_{2} u_{1}, u_{1} u_{2} u_{3}, u_{1} u_{3} u_{0}\right\}$ be a set of words, and $R_{f}=\left\{u_{1} u_{0} u_{3}\right\}, R_{f}^{(23)}=\left\{u_{1} u_{3} u_{0}\right\}$ are filters. First, apply the filter $R_{f}$

$$
R_{f} \circ_{23} R=\left\{u_{1} u_{0} u_{3}, u_{1} u_{1} u_{3}, u_{1} u_{2} u_{3}, u_{1} u_{3} u_{3}\right\} \text {. }
$$

Then apply the filter $R_{f}^{(23)}$

$$
R_{f}^{(23)} \circ_{23} R_{f} \circ_{23} R=\left\{u_{1} u_{0} u_{0}, u_{1} u_{1} u_{0}, u_{1} u_{2} u_{0}, u_{1} u_{3} u_{0}\right\} .
$$

Example 3 (crossover). Let $R=\left\{u_{1} u_{0} u_{0}, u_{1} u_{1} u_{0}, u_{1} u_{2} u_{1}, u_{1} u_{3} u_{2}\right\}$ be a population. Let us define the evolution operator $\mathrm{E}(R)=R \cup R \circ_{23} R$ and start a first step of evolution

$$
\mathrm{E}(R)=\left\{u_{1} u_{0} u_{0}, u_{1} u_{1} u_{0}, u_{1} u_{2} u_{0}, u_{1} u_{2} u_{1}, u_{1} u_{3} u_{1}, u_{1} u_{3} u_{2}\right\} .
$$

In example 4 we are going to denote 3-tuple $\left(u_{i_{1}}, u_{i_{2}}, u_{i_{3}}\right)$ as an implies $u_{i_{1}} \rightarrow\left(u_{i_{2}} \rightarrow u_{i_{3}}\right)$.

Example 4 (AI). Let $R=\left\{u_{1} \rightarrow\left(u_{1} \rightarrow u_{1}\right), u_{1} \rightarrow\left(u_{1} \rightarrow u_{2}\right)\right\}$ be a base set of AI premises. Let us define the semantic closure of $R$ as $[R]=\bigcup_{k=1}^{\infty}\left(R \cup R^{(23)}\right)^{k}$, where $R^{k+1}=R^{k} \circ_{23} R$ and $R^{1}=R$. By definition we have

$$
[R]=\left\{u_{1} \rightarrow\left(u_{1} \rightarrow u_{1}\right), u_{1} \rightarrow\left(u_{1} \rightarrow u_{2}\right), u_{1} \rightarrow\left(u_{2} \rightarrow u_{1}\right), u_{1} \rightarrow\left(u_{2} \rightarrow u_{2}\right)\right\} .
$$

Note that $\left(\left(u_{1} \rightarrow\left(u_{0} \rightarrow u_{3}\right)\right) \wedge\left(u_{1} \rightarrow\left(u_{2} \rightarrow u_{0}\right)\right)\right) \rightarrow\left(u_{1} \rightarrow\left(u_{2} \rightarrow u_{3}\right)\right)$ is tautology, so the inference rule $u_{1} \rightarrow\left(u_{0} \rightarrow u_{3}\right), u_{1} \rightarrow\left(u_{2} \rightarrow u_{0}\right) \dagger u_{1} \rightarrow\left(u_{2} \rightarrow u_{3}\right)$ preserves truth.

We also note that $\left(\left(\left(u_{1} \rightarrow u_{0}\right) \rightarrow u_{3}\right) \wedge\left(\left(u_{1} \rightarrow u_{2}\right) \rightarrow u_{0}\right)\right) \rightarrow\left(\left(u_{1} \rightarrow u_{2}\right) \rightarrow u_{3}\right)$ is tautology, too.

It makes perfect sense to use an indicator function $\chi_{R}: U^{n} \mapsto\{0,1\}$ for $R \in 2^{U^{n}}$, that is defined as

$$
\chi_{R}\left(u_{1}, . ., u_{n}\right)=\left\{\begin{array}{l}
1,\left(u_{1}, . ., u_{n}\right) \in R \\
0,\left(u_{1}, . ., u_{n}\right) \notin R
\end{array} .\right.
$$

In the case of finite set $U=\left\{u_{1}, . ., u_{m}\right\}$, we can use this function to define a join-vertices logical array $\psi^{R}:(1 . . m)^{n} \mapsto\{$ false,true $\}$ for (n-uniform) hypergraph. Let $f: 1 . . m \mapsto U$ be a total bijection and $R \in 2^{U^{n}}$. We define

$$
\psi_{k_{1}, . ., k_{n}}^{R}=\psi^{R}\left(k_{1}, . ., k_{n}\right)=\left\{\begin{array}{ll}
\text { true }, & \chi_{R}\left(f^{-1}\left(k_{1}\right), . ., f^{-1}\left(k_{n}\right)\right)=1 \\
\text { false, }, & \chi_{R}\left(f^{-1}\left(k_{1}\right), . ., f^{-1}\left(k_{n}\right)\right)=0
\end{array} .\right.
$$

Let us denote $\{$ false,true $\}$ as $D$ and a set of logical array defined above as $\mathrm{D}^{(1 . . m)^{n}}$. 
We also can set a logical algebra that generalized adjacency matrices algebra. In this way we define a binary operation $*_{i j}$ on $\mathrm{D}^{(1 . . m)^{n}}$

$$
\psi_{k_{1}, \ldots, k_{n}}^{1} *_{i j} \psi_{k_{1}, \ldots, k_{n}}^{2}=\bigvee_{s=1}^{m} \psi_{k_{1}, \ldots, k_{i-1}, s, k_{i+1}, \ldots, k_{j}, \ldots, k_{n}}^{1} \wedge \psi_{k_{1}, \ldots, k_{i}, \ldots, k_{j-1}, s, k_{j+1}, \ldots, k_{n}}^{2} .
$$

By our construction semigroups $\left\langle 2^{U^{n}},\left(\circ_{i j}\right)\right\rangle$ and $\left\langle D^{(1 . . m)^{n}},\left(*_{i j}\right)\right\rangle$ are isomorphic.

More interesting is the case of algebraic structures on an underlying set $\bigcup_{n=1}^{\infty} 2^{U^{n}}$ and operations from $2^{U^{n}}$ to $2^{U^{m}}$. For example, let us define the operations "gluing edges” $\circ_{g}$ and "replacing chains" $\circ_{r}$.

$$
\begin{gathered}
R_{1} \circ_{g} R_{2}=\left\{\left(u_{1}, . ., u_{m-1}, u_{2}^{\prime}, . ., u_{n}^{\prime}\right) \mid \exists u_{0}\left(u_{1}, . ., u_{m-1}, u_{0}\right) \in R_{1} \wedge\left(u_{0}, u_{2}^{\prime}, . ., u_{n}^{\prime}\right) \in R_{2}\right\}, \\
R_{1} \circ_{r} R_{2}=\left\{\left(u_{1}, . ., u_{i-1}, u_{j+1}^{\prime}, . ., u_{n}^{\prime}\right) \mid \exists u_{0} \exists i \exists j\left(u_{1}, . ., u_{i-1}, u_{0}, . ., u_{m}\right) \in R_{1} \wedge\left(u_{1}^{\prime}, ., u_{0}, u_{j+1}^{\prime}, . ., u_{n}^{\prime}\right) \in R_{2}\right\} .
\end{gathered}
$$

For the finitary relation $R=\left\{\left(u_{1}, u_{0}, u_{3}\right),\left(u_{1}, u_{2}, u_{0}\right)\right\}$ from Example 1 we can get

$$
\begin{gathered}
R^{(13)}=\left\{\left(u_{3}, u_{0}, u_{1}\right),\left(u_{0}, u_{2}, u_{1}\right)\right\}, \\
R \circ{ }_{g} R^{(13)}=\left\{\left(u_{1}, u_{0}, u_{0}, u_{1}\right),\left(u_{1}, u_{2}, u_{2}, u_{1}\right)\right\}, \\
R \circ_{r} R=\left\{\left(u_{1}\right),\left(u_{0}, u_{3}\right),\left(u_{1}, u_{0}\right),\left(u_{1}, u_{2}\right),\left(u_{1}, u_{3}\right),\left(u_{2}, u_{0}\right),\left(u_{1}, u_{2}, u_{3}\right)\right\} .
\end{gathered}
$$

It is clear that even in the case of finite set $U$ we would never make a finite representation for such algebraic structures. But in particular cases, maybe we can. This case is of interest.

\section{References}

[1] Hein M, Setzer S, Jost L and Rangapuram S S 2013 The total variation on hypergraphs-learning on hypergraphs revisited Advances in Neural Information Processing Systems 2427-2435

[2] Ricatte T, Gilleron R and Tommasi M 2014 Hypernode graphs for spectral learning on binary relations over sets Joint European Conference on Machine Learning and Knowledge Discovery in Databases 662-677

[3] Louis A 2015 Hypergraph markov operators, eigenvalues and approximation algorithms Proceedings of the Forty-Seventh Annual ACM on Symposium on Theory of Computing 713-722

[4] Zhang C, Hu S, Tang Z G and Chan T H 2017 Re-revisiting Learning on Hypergraphs: Confidence Interval and Subgradient Method Proceedings of the 34th International Conference on Machine Learning, in PMLR 70 4026-4034

[5] Pu L, Faltings B 2012 Hypergraph learning with hyperedge expansion Machine Learning and Knowledge Discovery in Databases 410-425

[6] Yu J, Tao D and Wang M 2012 Adaptive hypergraph learning and its application in image classification IEEE Transactions on Image Processing 21(7) 3262-3272

[7] Panagopoulos A, Wang C, Samaras D and Paragios N 2013 Simultaneous cast shadows, illumination and geometry inference using hypergraphs IEEE Transactions on Pattern Analysis and Machine Intelligence 35(2) 437-449

[8] Wang M, Wu X 2015 Visual classification by l1-hypergraph modeling IEEE Transactions on Knowledge and Data Engineering 27(9) 2564-2574

[9] Zhou D, Huang J and Scholkopf B 2007 Learning with Hypergraphs: Clustering, Classification, and Embedding Advances in Neural Information Processing Systems: Proceedings of the 2006 Conference 19 1601-1608

[10] Ghoshdastidar D, Ambedkar D 2014 Consistency of Spectral Partitioning of Uniform Hypergraphs under Planted Partition Model Advances in Neural Information Processing Systems 27: Annual Conference on Neural Information Processing Systems 397-405 
[11] Ghoshdastidar D, Ambedkar D 2015 A Provable Generalized Tensor Spectral Method for Uniform Hypergraph Partitioning Proceedings of the 32nd International Conference on Machine Learning, ICML 400-409

[12] Ghoshdastidar D, Ambedkar D 2017 Consistency of spectral hypergraph partitioning under planted partition model Ann. Statist. 45(1) 289-315

[13] Chien I, Lin C and Wang I 2018 Community Detection in Hypergraphs: Optimal Statistical Limit and Efficient Algorithms Proceedings of the Twenty-First International Conference on Artificial Intelligence and Statistics, in PMLR 84 871-879

[14] Mal’tsev A I 1973 Algebraic systems (Springer) p 319

[15] Chernov V M 2018 Ternary number systems in finite fields Computer Optics 42(4) 704-711 DOI: 10.18287/2412-6179-2018-42-4-704-711

[16] Tsvetov V P 2014 On a syntactic algorithm on graphs Proceedings of the International Conference Advanced Information Technologies and Scientific Computing (Samara, Russia) 235-238

[17] Tsvetov V P 2018 Dual ordered structures of binary relations Proceedings of the International Conference Information Technology and Nanotechnology. Session Data Science (Samara, Russia) 2635-2644 